DOUXAMI, Christine \& DEGAILlE, Philippe. - FESMAN

2010 : d'Est en Ouest, du Nord au Sud,

\title{
Alioune Sow
}

\section{OpenEdition}

\section{Journals}

Édition électronique

URL : http://journals.openedition.org/etudesafricaines/20904

DOI : 10.4000/etudesafricaines.20904

ISSN : $1777-5353$

\section{Éditeur}

Éditions de l'EHESS

\section{Édition imprimée}

Date de publication : 1 septembre 2017

Pagination : 766-769

ISBN : 978-2-7132-2686-1

ISSN : 0008-0055

\section{Référence électronique}

Alioune Sow, « douxamı, Christine \& degallle, Philippe. - FESMAN 2010 : d'Est en Ouest, du Nord au Sud, », Cahiers d'études africaines [En ligne], 227 | 2017, mis en ligne le 01 septembre 2017, consulté le 24 septembre 2020. URL : http://journals.openedition.org/etudesafricaines/20904 ; DOI : https://doi.org/ 10.4000/etudesafricaines.20904

Ce document a été généré automatiquement le 24 septembre 2020.

(C) Cahiers d'Études africaines 


\title{
DOUXAMI, Christine \& DEGAILLE, Philippe. - FESMAN 2010 : d'Est en Ouest, du Nord au Sud,
}

\author{
Alioune Sow
}

\section{RÉFÉRENCE}

DouXAMI, Christine \& DEGAILLE, Philippe. - FESMAN 2010 : d'Est en Ouest, du Nord au Sud, film documentaire (français et brésilien). Dakar, 108 min., 2010.

1 L'ambition du documentaire FESMAN 2010, réalisé par Christine Douxami et Philippe Degaille, est de faire découvrir la troisième édition du Festival mondial des arts nègres qui s'est tenu à Dakar du 10 au 31 décembre 2010 et dont le Brésil était l'invité d'honneur. Il est surtout consacré à une réflexion propice sur les enjeux d'un tel festival à travers un examen approfondi du thème de la « Renaissance africaine », initié par Thabo Mbeki et mobilisé par Abdoulaye Wade alors président du Sénégal, pour clôturer une année de commémorations du cinquantenaire des Indépendances africaines sur le continent. Les auteurs, en retrait et discrets, ont choisi de fonder l'ensemble de la réflexion sur la prise de parole des musiciens, dramaturges, comédiens, chorégraphes, plasticiens et organisateurs qui se confient à tour de rôle sur leur participation à l'événement mais aussi sur l'économie culturelle africaine, le crédit accordé au concept de "Renaissance africaine», la validité de l'idée de «panafricanisme » et la pertinence de la notion d'art nègre. De nombreuses images du projet remarqué de Senghor en 1966 ponctuent le documentaire. C'est donc l'occasion de revenir, dans une perspective comparatiste et à partir d'expériences et de performances contrastées et plurielles, sur le renouvellement de l'intervention artistique et de ses modalités en ce début du $\mathrm{xx}^{\mathrm{e}}$ siècle. C'est aussi une invitation à repenser les dynamiques culturelles du continent ainsi que dans les territoires 
diasporiques américains et européens et de vérifier la place du festival dans les imaginaires artistiques en Afrique et ailleurs.

2 Trois parties de durée inégale composent le documentaire. La première, intitulée «Postures des protagonistes » est courte. Sous forme de circuit découverte qui débute dans la périphérie de Dakar et s'achève sur le site du festival, elle permet à chaque intervenant de se présenter.

3 La seconde partie, "Rêves", se concentre sur la diaspora et aborde, à travers des entretiens avec des artistes brésiliens notamment, la question des perceptions et représentations de l'Afrique ainsi que ce qui distingue les participations au festival. La majorité des interventions confirme l'existence d'un « rapport affectif» (Chico César) avec le continent qui s'affirme par le sentiment d'être " en famille ", "à la maison " (Deborah Almeida), de retrouver à Dakar des sensations familières, kinesthésiques parfois: "des choses que ma mère faisait que j'ai retrouvées ici " (Neves). À ces relations s'ajoutent des observations sur la valeur épiphanique du festival dans la mesure où le séjour au Sénégal permet de remettre en question, voire de contredire, la condition noire au Brésil, déterminée par des formes de racialisation. Alors qu'au Brésil le Noir « est camouflé » (Chico César), au Sénégal il est « au centre » (Almeida). Pour les intervenants, ces déplacements obligent à penser autrement les termes de la définition identitaire : "Je suis Noir au Brésil et ici je suis métis » (Conceicao). Conjointement, le séjour et la participation défient les propositions et amalgames sur le métissage : « Être Noir c'est te différencier à l'intérieur du truc de métissage » (Chico César). Si certains témoignages semblent parfois teintés d'enchantement, ils n'en sont pas pour autant nostalgiques, ce qui demeure sans doute l'une des contributions majeures du documentaire. Ainsi, pour le musicien Chico César, le festival est appréhendé comme un véritable "rite de passage ", l'occasion pour lui d'affirmer les généalogies et de décrypter les protocoles qui fondent sa production musicale et qui procèdent de la combinaison entre « l'esthétique noire et le lien ancestral ».

4 Cette deuxième partie s'achève sur le rappel important que les énoncés diasporiques s'appuient sur le passé, sur le moment primordial de la séparation et de la Traite qui continue de "modeler les identités ». Cela est confirmé par les réflexions sur le lieu de mémoire du fort de l'île de Gorée dont les participants soulignent l'importance dans leur volonté de retrouver les traces d'une « ancestralité perdue » en reconnaissant avec émotion la «tristesse " et la "souffrance » (Daniel Lind Ramos) ressenties lors de la visite du mémorial.

5 Ces réflexions sur le passé conduisent à aborder le thème de la "renaissance " retenu par les organisateurs du festival. Ce terme, suggèrent les intervenants, devrait être reconsidéré, voire corrigé. Selon Garcia, l'interprétation contemporaine du mot est erronée car la renaissance est un processus qui, selon lui, remonte au moment de la Traite, lors de la traversée, à l'arrivée en terre étrangère, enclenchant des ajustements à un nouvel état et animant la « reconstruction et la réélaboration » par la diaspora.

6 La troisième partie, "Nous aussi ", est consacrée à cette problématique et soulève d'autres questions. La réflexion stimulante qu'elle propose inclut cette fois plusieurs thèmes et termes clés qui ont servi à la mise en place et à la promotion du festival : à la renaissance africaine s'ajoutent l'art nègre et le panafricanisme. Il résulte des entretiens une méfiance des artistes à l'égard de ces concepts déployés à chaque commémoration, rassemblement ou transition politique. Pour les uns, ces concepts sont « datés mais valides » (J.-F. Boclé). Pour les autres, ces paradigmes sont restrictifs : 
«Renaissance non. C'est une dynamique " (Alexis Peskine). Ils tendent à enfermer les artistes comme leur production dans des catégories immuables comme le soulève Georgina Asor Opuku lorsqu'elle entend la formule « art nègre » : « What is black ? It's world something... not black!»

Conscients de l'instrumentalisation politique qui a pu être faite de la mémoire du Fesman 1966, certains artistes tiennent néanmoins à préciser qu'ils en demeurent les principaux bénéficiaires et revendiquent la filiation avec l'école de pensée de la négritude qui l'a initié : «S'il n'y avait pas eu cette pensée on ne serait pas ici » (Olou). D'autres estiment qu'il est temps de relativiser ces liens et d'entreprendre de nouvelles prospections. Si la «dimension mythique » (Valérie Goma) de la relation entre Césaire, Damas, Senghor et les productions artistiques noires n'est plus à démontrer ni à remettre en cause, les échanges sur la créativité, ses dynamiques et ses modalités, montrent aussi la déception à constater que les productions artistiques contemporaines sont constamment ramenées à ces trois idées de renaissance, d'art nègre et de panafricanisme. Pour les artistes, il est temps de réactualiser ces termes en se reportant aux nombreuses formes inédites d'engagement à l'œuvre et de saisir enfin comment "l'Afrique [se prend] en main » (Kabengele Munanga). En s'appuyant sur les œuvres et leur esthétique, les artistes soulignent le renouvellement de la pensée en révélant l'émergence de formes et de pratiques artistiques inédites, marquées par la diversité et influencées par la mobilité. Ces formes et pratiques témoignent de relations insoupçonnées sur et avec le continent et révèlent par ailleurs les liens étroits maintenus entre la créativité et les transformations socio-politiques. Pour le chorégraphe Serge Coulibaly, suite aux démocratisations, "des chemins nouveaux " sont explorés et c'est la jeunesse qui s'en charge. C'est aussi ce qu'affirme brillamment le dramaturge Dorcy Rugamba qui, en prenant l'exemple du Rwanda et du génocide, montre comment le théâtre permet de dépasser les clivages et les postures victimaires : " fils de cette histoire qu'il faut exhumer » et " exorciser ", « on a plus envie de rester dans ces cases. Il faut un nouveau récit qui englobe toute une histoire». Ce qui caractérise ce "nouveau récit», ce sont ses versions et ses fragments ainsi que ses priorités multiples. Ainsi, pour l'acteur malien Bagayoko, ce récit prend, par exemple, la forme d'une narration introspective sur la période militaire afin de pouvoir résoudre les énigmes liées au passé et de déterminer les responsabilités individuelles et collectives. C'est la condition préalable à l'appréhension d'un futur serein : "L'avenir embarrasse celui qui ne connait pas le passé. »Ce récit se doit d'être inclusif pour « travailler sur l'espoir » (Coulibaly) et stimuler le « vivre ensemble » (Mamady Diffé ).

8 À ceux qui en douteraient encore, le documentaire FESMAN 2010 démontre de façon convaincante et à l'aide d'images et d'extraits de performances captivants (surtout dans la troisième partie), qu'il existe en Afrique et dans la diaspora, une créativité renouvelée et dynamique, des pratiques artistiques et culturelles fécondes et fédératrices. Au-delà de l'aspect pragmatique du festival « qui permet à la valorisation des cultures noires " (Modeste Nzapassara), on retiendra surtout les nouvelles pistes qui se dégagent des entretiens proposés, et qui se fondent sur les articulations diversifiées et novatrices, en partie affranchies des concepts ayant fait la négritude et l'art nègre. On retiendra aussi les nombreux croisements artistiques et intersections culturelles, élaborés par des artistes et des organisateurs résolus et animés par une volonté farouche de « lancer des ponts » (Younous Diallo). 